\title{
Active Disturbance Rejection Synchronization of Morris-Lecar Neurons
}

\author{
Wei Wei (D), Yanjie Shao $(\mathbb{D}$, and Min Zuo $\mathbb{D}$ \\ School of Computer and Information Engineering, Beijing Key Laboratory of Big Data Technology for Food Safety, \\ Beijing Technology and Business University, Beijing 100048, China \\ Correspondence should be addressed to Min Zuo; zuomin@btbu.edu.cn
}

Received 18 February 2018; Accepted 24 April 2018; Published 5 June 2018

Academic Editor: Zhile Yang

Copyright (C) 2018 Wei Wei et al. This is an open access article distributed under the Creative Commons Attribution License, which permits unrestricted use, distribution, and reproduction in any medium, provided the original work is properly cited.

\begin{abstract}
Synchronization of biological neurons is not only a hot topic, but also a difficult issue in the field of bioelectrical physiology. Numerous reported synchronization algorithms are designed on the basis of neural model, but they have deficiencies like relatively complex and poor robustness and are difficult to be realized. Morris-Lecar neuron is considered, and linear active disturbance rejection control (LADRC) is designed. Only one control input signal is utilized to synchronize membrane potentials of biological neurons. Meanwhile, in order to verify the robustness of synchronization, sinusoidal signal and parameter perturbations are introduced in numerical simulations. LADRC can still achieve satisfactory synchronization. Both theoretical and numerical simulation results show that LADRC is capable of estimating and cancelling disturbances and uncertainties. Neither accurate neural models nor concrete disturbance signal models are indispensable. A more practical and effective thought is provided to address the synchronization between neurons.
\end{abstract}

\section{Introduction}

Complex and powerful biological nervous systems are composed of a great number of connected neurons. By organizing and analysing experimental data of membrane potentials of biological neurons, it is found that synchronization of neuronal membrane potentials plays a crucial role in biological information processing and transmission. The synchronization and synchronized degree between biological neurons are vital for physiological function [1]. Actualizing the synchronization of biological neurons has become a hot and difficult topic in bioelectricity physiology, nonlinear system theory, control, and many other areas.

For quantitative analysis of discharging characteristics, Hodgkin-Huxley $(\mathrm{HH})$ model [2] was proposed by Hodgkin and Huxley in 1950s. It is the first mathematic model that describes dynamics of biological neuronal membrane potentials, which has turned into a milestone of bioelectric physiology. Subsequently, various neuronal models describing different biological neuronal discharging modes have been proposed, such as FitzHugh-Nagumo (FHN) model [3],
Ghostburster model [4], Hindmarsh-Rose (HR) model [5], and Chay model [6].

Based on the above models, in order to assist the abnormal nervous systems to operate normally, scholars from nonlinear control area began to study how to exert an external control signal, i.e., an external electrical stimulation, to drive different discharging modes between neurons to be synchronized. A variety of quantitative analyses, control, and synchronization studies have been carried out. For instance, according to $\mathrm{HH}$ neuron model, accurate feedback [7] has been applied to realize the discharging synchronization between two $\mathrm{HH}$ neurons; under assumptions that all states are available, nonlinear control is proposed [8], additionally, when only membrane potential is available, linear adaptive control has also been designed to realize the discharging synchronization of HR neurons [8]; when system states and model are known, stable feedback control [9] based on Lyapunov stability theory has been designed and the synchronization of FitzHugh-Nagumo (FHN) neurons is achieved; adaptive neural network $H_{\infty}$ approach proposed in [10] is utilized to synchronize two Ghostburster neurons. 
TABLE 1: Variables of a Morris-Lecar neuron.

\begin{tabular}{lc}
\hline Variables & Values \\
\hline$C$ & 5 \\
$g_{L}$ & 2 \\
$V_{L}$ & -60 \\
$g_{\mathrm{Ca}}$ & 4 \\
$V_{\mathrm{Ca}}$ & 120 \\
$g_{\mathrm{K}}$ & 8 \\
$v_{\mathrm{K}}$ & -80 \\
$\phi$ & $1 / 15$ \\
$v_{1}$ & -1.2 \\
$v_{2}$ & 18 \\
$v_{3}$ & 2 \\
$v_{4}$ & 17.4 \\
\hline
\end{tabular}

Morris-Lecar neuron is a kind of simplified biological neuron model, its asymptotic behaviour has been analysed in $[11,12]$. González has explained the pacemaker dynamics in special parameter space [13]. For the purpose of describing memory features of Morris-Lecar neuron, fractal dimension Morris-Lecar neuron model has also been proposed in [14]. In order to achieve the synchronization of Morris-Lecar neurons, linear feedback control [12], adaptive $H_{\infty}$ control [15], and other control algorithms have been reported, respectively.

Actually, two points are of great importance in the synchronization of neurons by external stimulations. Firstly, the synchronization approach should be robust enough to disturbance. Owing to the weakness of electrical signals created by neurons, the neuronal membrane potentials are sensitive to disturbance. Model based control, whose performance largely depends on the controlled system model, cannot keep the satisfactory performance in presence of disturbance. Therefore, control algorithms which are robust enough to disturbance are able to guarantee the synchronization. Secondly, low power electrical stimulations play a critical role in the synchronization [16]. As we know, a high power electrical stimulation is harmful to biological tissues. Therefore, reducing the power of a stimulation will guarantee the safe of tissues and lengthen the lifetime of neurological implant.

Active disturbance rejection control (ADRC) is the very kind of control, which has strong ability to reject disturbance. By rethinking the essence of control and making a discussion on control theory (i.e., a model analysis approach or a direct control approach?) Han clarified the nature of control [17] in the late 1980s and proposed ADRC [18]. It does not need faithful model information. Before disturbance corrupting system output, ADRC estimates and cancels disturbance to guarantee the system output to be still desired. Therefore, in presence of disturbance, less oscillations of a control signal are needed to regulate system output. In other words, less power is consumed in regulation. So far, theoretical and applied researches on ADRC have been becoming a hot topic and numerous results have been reported in such area [19-21].

However, for ADRC, many parameters have to be determined. Performance of a closed-loop system, to a great extent, depends on the experience of tuning parameters. It does limit the applications of ADRC. In order to reduce difficulty of parameter tuning, Gao proposed linear active disturbance rejection control (LADRC) and provided bandwidthparameterization based tuning method for LADRC [22].

In this paper, the synchronization of Morris-Lecar neuronal membrane potentials is considered and LADRC is utilized to reject disturbance and achieve desired synchronization. The rest of the paper is organized as follows: problem description is presented in Section 2, basic LADRC and the design of linear active disturbance rejection synchronization are provided in Section 3, simulation results are given in Section 4, and, finally, the concluding remarks are given in Section 5.

\section{Problem Description}

2.1. Morris-Lecar Neuron Model. Morris-Lecar neuron model can be expressed as follows [12]:

$$
\begin{aligned}
C \frac{d V}{d t}= & I_{\text {ext }}-g_{L}\left(V-V_{L}\right)-g_{\mathrm{Ca}} \beta(V)\left(V-V_{\mathrm{Ca}}\right) \\
& -g_{\mathrm{K}} n\left(V-V_{\mathrm{K}}\right) \\
\frac{d n}{d t}= & \tau(V)(\alpha(V)-n),
\end{aligned}
$$

where

$$
\begin{aligned}
& \alpha(V)=0.5\left(1+\tanh \left(\frac{\left(V-v_{3}\right)}{v_{4}}\right)\right), \\
& \beta(V)=0.5\left(1+\tanh \left(\frac{\left(V-v_{1}\right)}{v_{2}}\right)\right), \\
& \tau(V)=\phi \cosh \left(\frac{\left(V-v_{3}\right)}{v_{4}}\right)
\end{aligned}
$$

$V$ is the membrane potential, $I_{\text {ext }}$ is the externally applied DC current, $t$ denotes the time measured in milliseconds, $n$ is the activation variable for potassium, $V_{\mathrm{K}}, V_{\mathrm{Ca}}, V_{L}$ are equilibrium potentials of potassium, calcium, and leakage currents, respectively. $g_{\mathrm{K}}, g_{\mathrm{Ca}}, g_{L}$ represent corresponding maximum conductance of corresponding ionic currents and $C$ stands for membrane capacitance. $v_{1}$ is the midpoint potential at which the calcium current is half-activated, $v_{2}$ is a constant that corresponds to the steepness of the activation voltage dependence, $v_{3}$ is the activation midpoint potential at which the potassium current is half-activated, and $v_{4}$ denotes the slope factor of the potassium activation. $\phi$ represents a constant. Here, values of each variable are listed in Table 1.

2.2. Discharge Characteristic of a Morris-Lecar Neuron. Neuron owns different discharge characteristics according to different values of external electric fields. Discharge characteristics of a Morris-Lecar neuron, when $I_{\text {ext }}=50$ and $I_{\text {ext }}=$ 200, are shown in Figure 1, respectively.

2.3. Synchronization of Morris-Lecar Neurons. Master and slave Morris-Lecar neurons are defined as follows:

$$
\frac{d V_{m}}{d t}=\left[I_{\mathrm{ext} m}-g_{L}\left(V_{m}-V_{L}\right)-g_{\mathrm{Ca}} \beta\left(V_{m}\right)\left(V_{m}-V_{\mathrm{Ca}}\right)\right.
$$




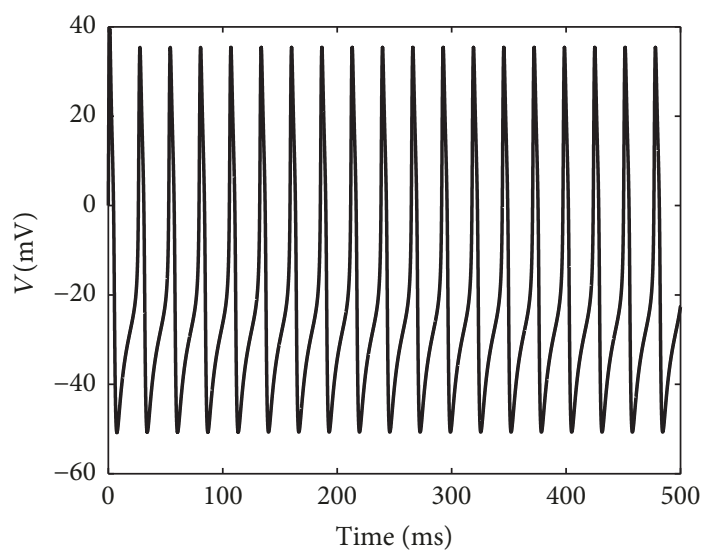

(a) $I_{\text {ext }}=50$

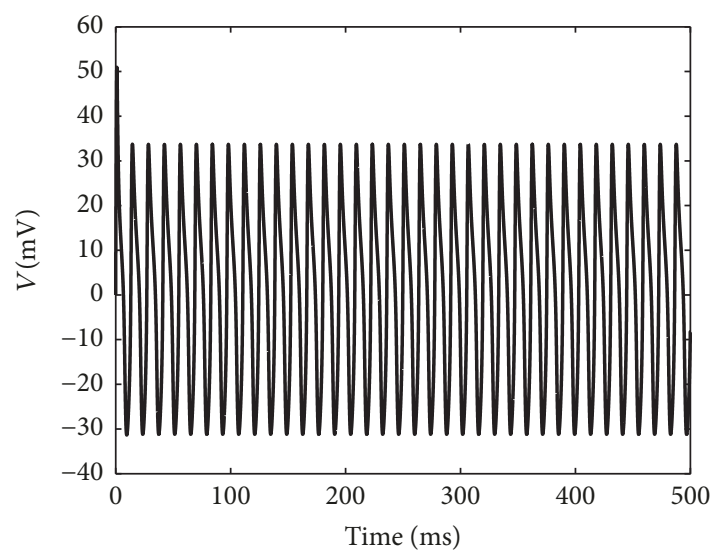

(b) $I_{\text {ext }}=200$

Figure 1: Membrane potential of a Morris-Lecar neuron when $I_{\text {ext }}$ are different.

$$
\begin{aligned}
& \left.-g_{\mathrm{K}} n_{m}\left(V_{m}-V_{\mathrm{K}}\right)\right] \frac{1}{C} \\
& \frac{d n_{m}}{d t}=\tau\left(V_{m}\right)\left(\alpha\left(V_{m}\right)-n_{m}\right), \\
& \frac{d V_{s}}{d t}=\frac{1}{C}\left[I_{\mathrm{exts}}-g_{L}\left(V_{s}-V_{L}\right)-g_{\mathrm{Ca}} \beta\left(V_{s}\right)\left(V_{s}-V_{\mathrm{Ca}}\right)\right. \\
& \left.-g_{\mathrm{K}} n_{s}\left(V_{s}-V_{\mathrm{K}}\right)\right]+u, \\
& \frac{d n_{s}}{d t}=\tau\left(V_{s}\right)\left(\alpha\left(V_{s}\right)-n_{s}\right),
\end{aligned}
$$

where footnotes $m$ and $s$ represent the master neuron and the slave neuron, respectively. $u$ is the external control input, i.e., the external electrical stimulation, which drives the slave neuron to act the same as the master neuron.

In this paper, the synchronization of Morris-Lecar neuronal membrane potentials is to realize $\lim _{t \rightarrow \infty}\left(V_{m}-V_{s}\right)=0$ by LADRC.

\section{Linear Active Disturbance Rejection Synchronization}

3.1. Brief Introduction to Linear Active Disturbance Rejection Control. In this paper, second-order linear active disturbance rejection control is designed to realize the synchronization. Assuming that a first-order unknown nonlinear controlled system can be described as

$$
\dot{y}=f(y, \dot{y}, w)+b u
$$

where $y$ is the system output, $w$ is external disturbance, $f$ denotes unknown system dynamics, $b$ is the control coefficient, and $u$ is the control input signal. Here, LADRC is utilized, and the control input can be designed as

$$
u=\frac{k_{p}\left(y_{r}-z_{1}\right)-z_{2}}{b_{0}}
$$

where $k_{p}$ is the proportional coefficient, $y_{r}$ is the desired output, $b_{0}$ is a tunable parameter, $z_{1}, z_{2}$ are outputs of the extended state observer (ESO), and ESO is designed as

$$
\begin{aligned}
& \dot{z}_{1}=z_{2}+l_{1}\left(y-z_{1}\right)+b_{0} u, \\
& \dot{z}_{2}=l_{2}\left(y-z_{1}\right),
\end{aligned}
$$

where $z_{1}$ estimates the system output $y, z_{2}$ estimates the integrated disturbance including internal uncertainties and unmodeled dynamics, and external disturbances, $y$ is the system output, $l_{1}, l_{2}$ are gains of ESO, and $u$ is the control input defined in (5).

If we let $\mathbf{z}=\left[z_{1}, z_{2}\right]^{T}, e_{o}=y-z_{1}$, then extended state observer (6) can be rewritten as

$$
\dot{\mathbf{z}}=\mathbf{A}_{o} \mathbf{z}+\mathbf{B}_{o} u+\mathbf{L} e_{o},
$$

where

$$
\begin{aligned}
\mathbf{A}_{o} & =\left[\begin{array}{ll}
0 & 1 \\
0 & 0
\end{array}\right], \\
\mathbf{B}_{o} & =\left[\begin{array}{l}
0 \\
b_{0}
\end{array}\right], \\
\mathbf{L} & =\left[\begin{array}{l}
l_{1} \\
l_{2}
\end{array}\right] .
\end{aligned}
$$

For the first-order unknown nonlinear controlled system, it can be rewritten as

$$
\begin{aligned}
\dot{y} & =f(y, \dot{y}, w)+b u \\
& =f(y, \dot{y}, w)+\left(b-b_{0}\right) u+b_{0} u \\
& \triangleq \bar{f}+b_{0} u .
\end{aligned}
$$

Here, $\bar{f}$ is defined as $\bar{f} \triangleq f(y, \dot{y}, w)+\left(b-b_{0}\right) u$, which represents the integrated disturbance. 


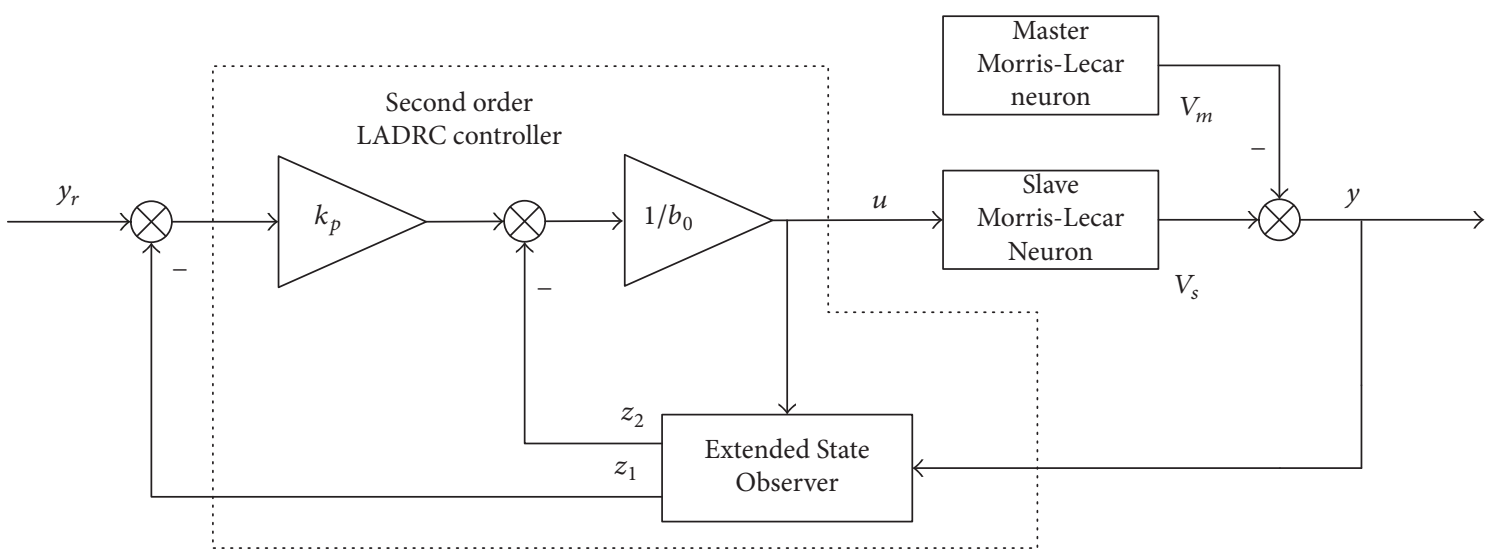

FIGURE 2: Synchronization structure of Morris-Lecar neurons by LADRC.

According to control input (5), when ESO performs well, i.e., $z_{1} \approx y, z_{2} \approx \bar{f}$, we have the closed-loop system as

$$
\begin{aligned}
\dot{y} & =\bar{f}+b_{0} u \\
& =\bar{f}+k_{p}\left(y_{r}-z_{1}\right)-z_{2} \\
& \approx k_{p}\left(y_{r}-z_{1}\right) .
\end{aligned}
$$

Here, a nonlinear controlled system, by LADRC, is approximately linearized. By setting proper parameters, one can get the desired performance.

\subsection{Closed-Loop Stability of Linear Active Disturbance Rejec-} tion Synchronization. LADRC is taken to realize the synchronization of Morris-Lecar neurons; synchronization structure of Morris-Lecar neurons is given in Figure 2.

In Figure 2, $V_{m}$ is the membrane potential of the master neuron, and $V_{s}$ is the membrane potential of the slave neuron, respectively. Let $\mathbf{x}_{1}=\left[V_{m}, n_{m}\right]^{T}, \mathbf{x}_{2}=\left[V_{s}, n_{s}\right]^{T}$, system model of the master and slave neurons can be rewritten as

$$
\begin{aligned}
& \dot{\mathbf{x}}_{1}=\mathbf{A} \mathbf{x}_{1}+\mathbf{F}\left(\mathbf{x}_{1}\right), \\
& \dot{\mathbf{x}}_{2}=\mathbf{A} \mathbf{x}_{2}+\mathbf{F}\left(\mathbf{x}_{2}\right)+\mathbf{B} u,
\end{aligned}
$$

where

$$
\begin{aligned}
& \mathbf{A}=\left[\begin{array}{cc}
\frac{-g_{L}}{C} & 0 \\
0 & 0
\end{array}\right], \\
& \mathbf{B}=\left[\begin{array}{l}
1 \\
0
\end{array}\right], \\
& \mathbf{F}\left(\mathbf{x}_{1}\right)=\left[\begin{array}{l}
f_{1}\left(\mathbf{x}_{1}\right) \\
f_{2}\left(\mathbf{x}_{1}\right)
\end{array}\right], \\
& \mathbf{F}\left(\mathbf{x}_{2}\right)=\left[\begin{array}{l}
f_{1}\left(\mathbf{x}_{2}\right) \\
f_{2}\left(\mathbf{x}_{2}\right)
\end{array}\right],
\end{aligned}
$$

$$
\begin{aligned}
& f_{1}\left(\mathbf{x}_{1}\right) \\
& =\frac{I_{\mathrm{ext} m}+g_{L} V_{L}-g_{\mathrm{Ca}} \beta\left(V_{m}\right)\left(V_{m}-V_{\mathrm{Ca}}\right)-g_{\mathrm{K}} n_{m}\left(V_{m}-V_{\mathrm{K}}\right)}{C}, \\
& f_{1}\left(\mathbf{x}_{2}\right) \\
& =\frac{I_{\mathrm{exts}}+g_{L} V_{L}-g_{\mathrm{Ca}} \beta\left(V_{s}\right)\left(V_{s}-V_{\mathrm{Ca}}\right)-g_{\mathrm{K}} n_{s}\left(V_{s}-V_{\mathrm{K}}\right)}{C}, \\
& f_{2}\left(\mathbf{x}_{1}\right)=\tau\left(V_{m}\right)\left(\alpha\left(V_{m}\right)-n_{m}\right), \\
& f_{2}\left(\mathbf{x}_{2}\right)=\tau\left(V_{s}\right)\left(\alpha\left(V_{s}\right)-n_{s}\right) .
\end{aligned}
$$

Consider the disturbance, system (11) can be rewritten as

$$
\begin{aligned}
& \dot{\mathbf{x}}_{1}=\mathbf{A} \mathbf{x}_{1}+\mathbf{F}\left(\mathbf{x}_{1}\right), \\
& \dot{\mathbf{x}}_{2}=\mathbf{A} \mathbf{x}_{2}+\mathbf{F}\left(\mathbf{x}_{2}\right)+\mathbf{B} d+\mathbf{B} u,
\end{aligned}
$$

where $d$ is bounded disturbance signal.

Let $\mathbf{e}_{c}=\mathbf{x}_{2}-\mathbf{x}_{1}=\left[\begin{array}{l}e_{v} \\ e_{n}\end{array}\right]=\left[\begin{array}{l}V_{s}-V_{m} \\ n_{s}-n_{m}\end{array}\right]$ be synchronization error vector, and synchronization error system of MorrisLecar neuronal membrane potentials can be obtained from (13):

$$
\dot{\mathbf{e}}_{c}=\mathbf{A} \mathbf{e}_{c}+\mathbf{F}\left(\mathbf{x}_{2}\right)-\mathbf{F}\left(\mathbf{x}_{1}\right)+\mathbf{B} d+\mathbf{B} u
$$

i.e.,

$$
\begin{aligned}
& \dot{e}_{v}=\frac{-g_{L}}{C} e_{v}+f_{1}\left(\mathbf{x}_{2}\right)-f_{1}\left(\mathbf{x}_{1}\right)+d+u, \\
& \dot{e}_{n}=f_{2}\left(\mathbf{x}_{2}\right)-f_{2}\left(\mathbf{x}_{1}\right) .
\end{aligned}
$$

The first synchronization error equation in (15) can be written as

$$
\begin{aligned}
\dot{e}_{v} & =\frac{-g_{L}}{C} e_{v}+f_{1}\left(\mathbf{x}_{2}\right)-f_{1}\left(\mathbf{x}_{1}\right)+d+u \\
& =\frac{-g_{L}}{C} e_{v}+f_{1}\left(\mathbf{x}_{2}\right)-f_{1}\left(\mathbf{x}_{1}\right)+d+\left(1-b_{0}\right) u+b_{0} u \\
& =G\left(e_{v}, \mathbf{x}_{1}, \mathbf{x}_{2}, u, d\right)+b_{0} u,
\end{aligned}
$$


where $G\left(e_{v}, \mathbf{x}_{1}, \mathbf{x}_{2}, u, d\right)$ can be regarded as the integrated disturbance that includes internal uncertainties, un-modeled dynamics, and external uncertainties.

From Figure 2, we can see that $y=V_{s}-V_{m}$. The output $z_{1}$ of ESO estimates $y=V_{s}-V_{m}$, i.e., the synchronization error $e_{v}$, and $z_{2}$ estimates integrated disturbance $G(\bullet)$.

Let $h=\dot{G}(\bullet)$, and then

$$
\begin{aligned}
\dot{e}_{v} & =G\left(e_{v}, \mathbf{x}_{1}, \mathbf{x}_{2}, u, d\right)+b_{0} u, \\
\dot{G}(\cdot) & =h .
\end{aligned}
$$

Let $\boldsymbol{\varepsilon}=\left[\begin{array}{l}\varepsilon_{1} \\ \varepsilon_{2}\end{array}\right]=\left[\begin{array}{l}e_{v}-z_{1} \\ G-z_{2}\end{array}\right]$ be observation error of ESO; then observation error equation of ESO can be obtained by subtracting system (6) from system (17); i.e.,

$$
\dot{\boldsymbol{\varepsilon}}=\mathbf{A}_{\varepsilon} \boldsymbol{\varepsilon}+\mathbf{E} h,
$$

where

$$
\begin{aligned}
\mathbf{A}_{\varepsilon} & =\left[\begin{array}{ll}
-l_{1} & 1 \\
-l_{2} & 0
\end{array}\right], \\
\mathbf{E} & =\left[\begin{array}{l}
0 \\
1
\end{array}\right] .
\end{aligned}
$$

Lemma 1 (see [23]). If $h$ is globally Lipschitz on $\mathbf{x}_{1}, \mathbf{x}_{2}$, then extended state observer gain $\mathbf{L}=\left[l_{1}, l_{2}\right]^{T}$ can be selected to make observation errors converge to zero asymptotically; i.e., $\lim _{t \rightarrow \infty} \varepsilon_{1}=\lim _{t \rightarrow \infty} \varepsilon_{2}=0$.

Lemma 2 (see [23]). If observation errors of the extended state observer are asymptotically convergent to zero, and then there exist appropriate parameters, for the closed-loop system, which can force the synchronization error to converge to zero asymptotically.

According to Lemmas 1 and 2, we have the following theorem.

Theorem 3. If $h$ is globally Lipschitz on $\mathbf{x}_{1}, \mathbf{x}_{2}$, appropriate parameters of controller and extended state observer, i.e., $k_{p}$ and $\mathbf{L}=\left[l_{1}, l_{2}\right]^{T}$, can be chosen so as to make synchronization error system (15) be asymptotically stable; i.e., asymptotic synchronization of the membrane potentials can be realized.

Proof. Substitute linear active disturbance rejection control law (5) into system (16); considering $y_{r}=0$ in synchronization, we have

$$
\begin{aligned}
\dot{e}_{v} & =G\left(e_{v}, \mathbf{x}_{1}, \mathbf{x}_{2}, u, d\right)+b_{0} u \\
& =-k_{p} z_{1}+\varepsilon_{2} \\
& =-k_{p} e_{v}+k_{p} \varepsilon_{1}+\varepsilon_{2} ;
\end{aligned}
$$

i.e.

$$
\dot{e}_{v}=-k_{p} e_{v}+k_{p} \varepsilon_{1}+\varepsilon_{2} .
$$

Choosing suitable ESO gain vector $\mathbf{L}=\left[l_{1}, l_{2}\right]^{T}$, one can make observation errors converge to zero. At the same time, suitable control parameter $k_{p}$ will drive the tracking error (21) to converge to zero asymptotically; i.e., synchronization error between $V_{s}$ and $V_{m}$ is asymptotic to zero.

When $e_{v}=V_{s}-V_{m}=0$, i.e., $V_{m}=V_{s}$, we have

$$
\begin{aligned}
\dot{e}_{n} & =f_{2}\left(\mathbf{x}_{2}\right)-f_{2}\left(\mathbf{x}_{1}\right) \\
& =\tau\left(V_{s}\right)\left(\alpha\left(V_{s}\right)-n_{s}\right)-\tau\left(V_{m}\right)\left(\alpha\left(V_{m}\right)-n_{m}\right) \\
& =\tau\left(V_{m}\right)\left(\alpha\left(V_{m}\right)-n_{s}-\alpha\left(V_{m}\right)+n_{m}\right) \\
& =\tau\left(V_{m}\right)\left(n_{m}-n_{s}\right) \\
& =-\tau\left(V_{m}\right) e_{n} .
\end{aligned}
$$

Since $\tau(V)=\phi \cosh \left(\left(V-v_{3}\right) / v_{4}\right)$, then

$$
\tau(V)=\phi \cosh (\cdot)=\phi \frac{\exp (\cdot)+\exp ^{-1}(\cdot)}{2} \geq \phi>0 .
$$

Hence, the zero dynamic of synchronization error system (15) is also asymptotically stable.

Therefore, selecting suitable ESO gain vector $\mathbf{L}=\left[l_{1}, l_{2}\right]^{T}$ and control parameter $k_{p}$, one can synchronize the membrane potentials of Morris-Lecar neurons asymptotically.

\section{Simulation Studies}

In order to verify LADRC in the synchronization of MorrisLecar neurons, initial conditions are chosen the same as the ones taken in [12], and three groups of simulations are performed. In the first group, no disturbance is considered, and synchronization performance has been compared. In the second group, numerical results have been compared, and the synchronization of LADRC has been confirmed in presence of sinusoidal disturbance. In the last group, parameters of slave neurons are changed to simulate parameter perturbations of a controlled plant. Results also verify LADRC.

Parameters of LADRC are chosen according to the approach proposed in [22]. Let control bandwidth $\omega_{c}$, bandwidth of ESO $\omega_{o}$, and control parameter $k_{p}$ satisfy $k_{p}=\omega_{c}$, $\omega_{o}=10 \omega_{c}$.

Group I. Synchronization is achieved in absence of disturbance. Control parameters and integral of absolute error (IAE, which can be calculated as $\int\left|e_{v}\right| d t$ ) are present in Table 2.

In Table $2, k_{1}, k_{2}$ are the same values chosen as those given in [12]. $\mathrm{IAE}_{1}$ is the IAE value generated by controllers taken in [12], $\mathrm{IAE}_{0}$ is the IAE value produced by LADRC. $\omega_{c}, b_{0}$ are parameters of LADRC. Synchronous response and comparisons of control inputs are shown in Figure 3. Figure 3(a) presents the synchronous response of LADRC. Figure 3(b) exhibits the comparison of synchronization errors between controllers presented in [12] and LADRC. In Figure 3(b), $e_{v}$ is the synchronization error generated by LADRC, while $e_{v 1}$ represents synchronization error generated by controllers proposed in [12]. Figure 3(c) is the comparison of $e_{n}$, which is produced by LADRC, and $e_{n 1}$, which is generated by controllers utilized in [12]. Figure 3(d) is the comparisons among 
TABLE 2: Control parameters and IAE values (in absence of disturbance).

\begin{tabular}{lccc}
\hline Control parameters & Values & IAE indexes & Values \\
\hline$k_{1}$ & 20 & IAE $_{1}$ & 54.8163 \\
$k_{2}$ & 20 & & \\
$\omega_{c}$ & 26 & IAE $_{0}$ & 8.6921 \\
$b_{0}$ & -50 & & \\
\hline
\end{tabular}
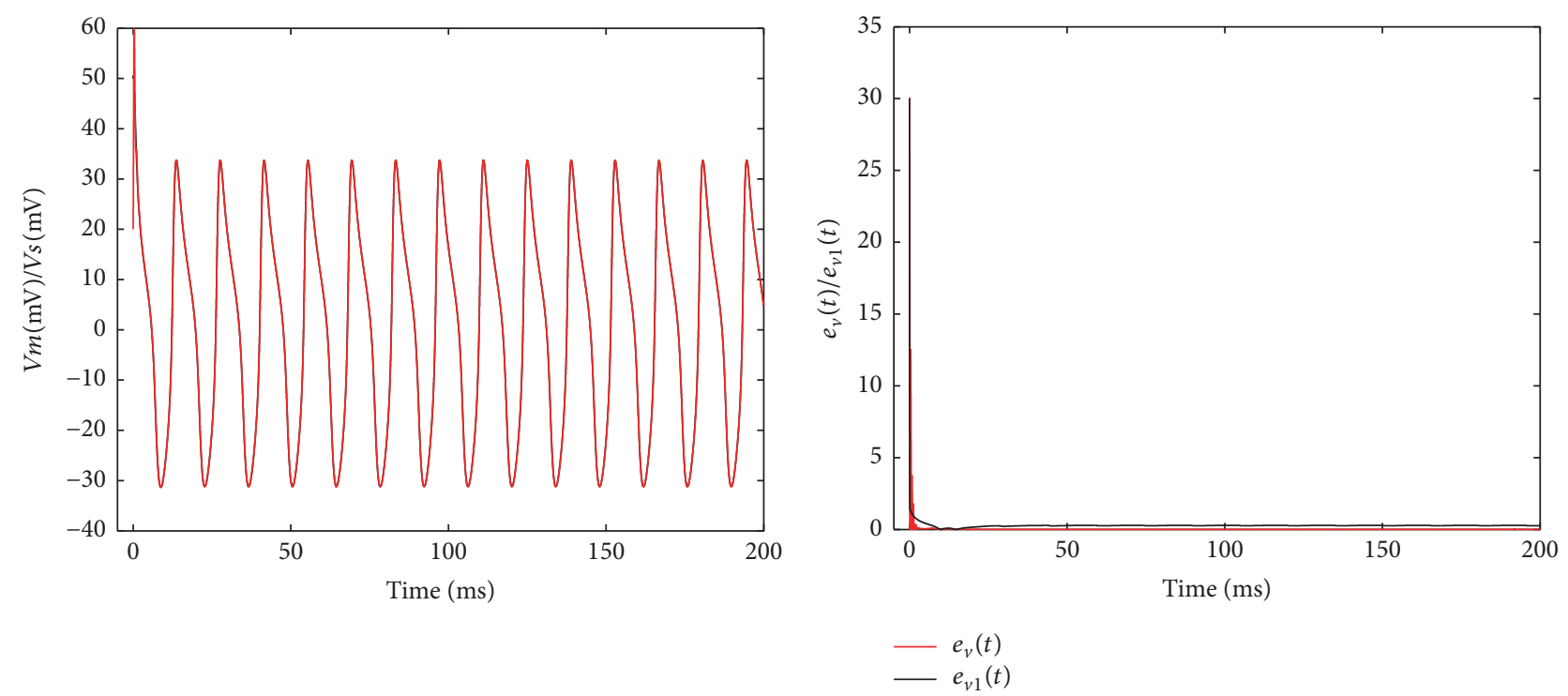

(a)
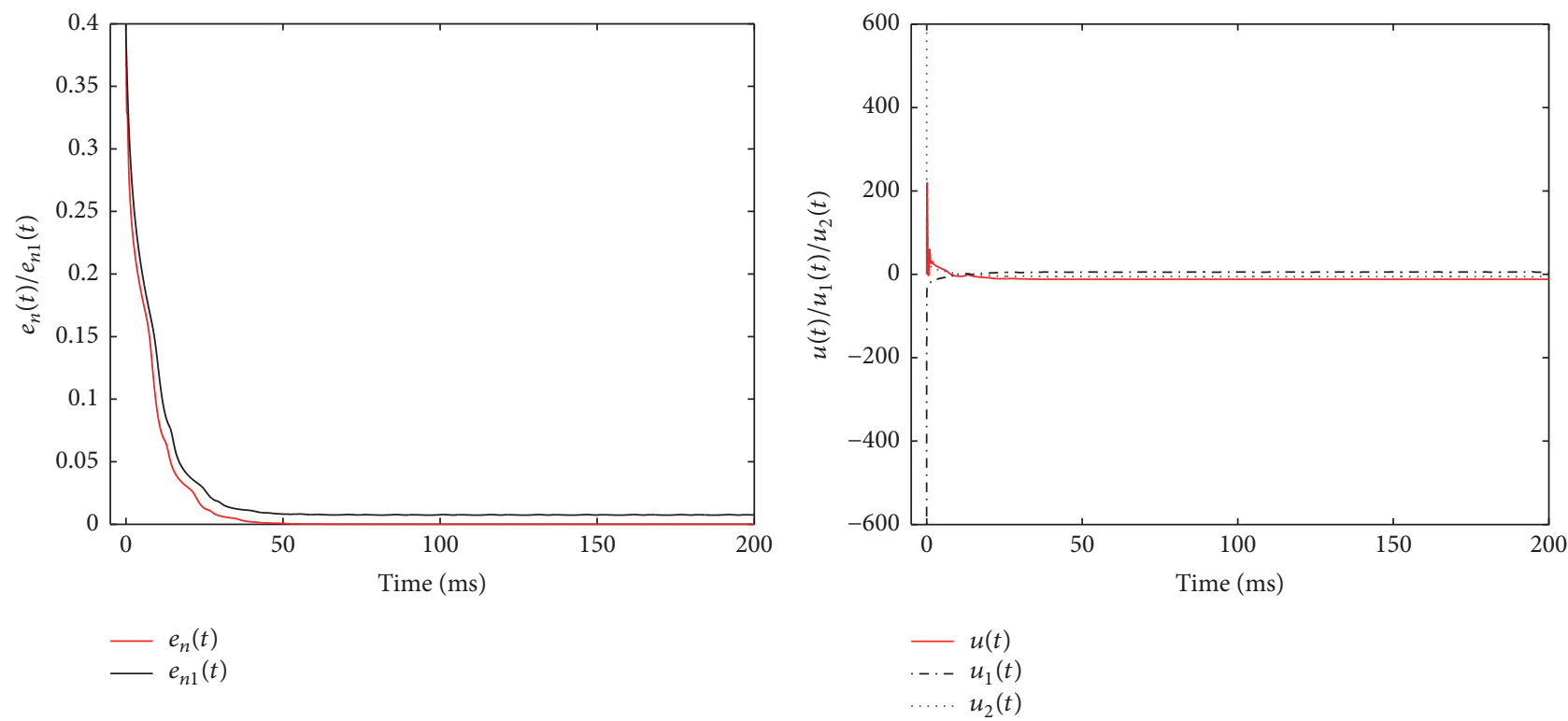

(c)

(d)

FIGURE 3: Synchronous performance of Morris-Lecar neurons and the comparison of control inputs (in absence of disturbance).

control input $u$ of LADRC and two control inputs $u_{1}$ and $u_{2}$ designed in [12].

From Table 2 and Figure 3, it can be seen that LADRC, compared with two controllers used in [12], is able to get smaller synchronization error and IAE values with less control energy.
Group II. For the purpose of verifying synchronization in presence of disturbance, sinusoidal disturbance $(d(t)=$ $10 \sin t)$ is taken. Synchronizations have been compared between controllers designed in [12] and LADRC. Controller parameters and IAE values are shown in Table 3. 
TABLE 3: Control parameters and IAE values (in presence of sinusoidal disturbance).

\begin{tabular}{lccc}
\hline Control parameters & Values & IAE indexes & Values \\
\hline$k_{1}$ & 20 & $\mathrm{IAE}_{1}$ & 275.2547 \\
$k_{2}$ & 20 & & $\mathrm{IAE}_{0}$ \\
$\omega_{c}$ & 26 & & 21.0479 \\
$b_{0}$ & -50 & \\
\hline
\end{tabular}

TABLE 4: Control parameters and IAE values (in presence of parameter perturbations).

\begin{tabular}{lccc}
\hline Control parameters & Values & IAE indexes & Values \\
\hline$k_{1}$ & 20 & $\mathrm{IAE}_{1}$ & 259.0081 \\
$k_{2}$ & 20 & & \\
$\omega_{c}$ & 26 & $\mathrm{IAE}_{0}$ & 22.7972 \\
$b_{0}$ & -50 & & \\
\hline
\end{tabular}

In Table $3, k_{1}, k_{2}, \omega_{c}, b_{0}$ are the same values taken as those given in Table 2. $\mathrm{IAE}_{1}$ and $\mathrm{IAE}_{0}$ are IAE values generated by controller taken in [12] and LADRC, respectively. Control is activated at $200 \mathrm{~ms}$, and the sinusoidal disturbance signal is introduced from $400 \mathrm{~ms}$. Simulation lasts $600 \mathrm{~ms}$. Synchronization response and the comparisons are given in Figure 4.

Figure 4(a) presents trajectories of neurons as time passes; Figure 4(b) is the phase trajectories between $V m$ and $V s$. It will be a line when synchronization is achieved. Obviously, Figure 4(b) shows that synchronization is obtained. Figures 4(c), 4(d), and 4(e) and IAE values given in Table 3 depict the fact that, with less control energy, smaller synchronization errors and IAE values can be obtained by LADRC. Simulation results manifest that ESO can estimate the disturbance signal effectively and then disturbance will be compensated to guarantee satisfactory synchronization.

Group III. For the purpose of verifying synchronization, parameters of slave neurons have also been changed. According to $[13], g_{\mathrm{Ca}}, g_{\mathrm{K}}$ can be chosen from $[0,12]$ and $[0,24]$, respectively. In simulations, $g_{\mathrm{Ca}}, g_{\mathrm{K}}$ of slave neurons have been changed by $100 \%$; i.e., $g_{\mathrm{Ca}}=8$ and $g_{\mathrm{K}}=16$, from $400 \mathrm{~ms}$ to the end of simulation. Controller parameters and IAE values are listed in Table 4.

In Table $4, k_{1}, k_{2}, \omega_{c}, b_{0}$ are the same values taken as those listed in Table 3. Control is also activated from $200 \mathrm{~ms}$, and the changed parameters (see Figure 5(f)) are introduced from $400 \mathrm{~ms}$. Simulation lasts $600 \mathrm{~ms}$. Synchronization response and the comparisons are given in Figure 5.

Figure 5 shows that when $g_{\mathrm{Ca}}$ and $g_{\mathrm{K}}$ are changed, smaller synchronization errors can be achieved by LADRC. Both Figure 5 and IAE values given in Table 4 show the same fact that, with the help of ESO, LADRC is able to get better synchronization.

Numerical results show that LADRC is able to achieve desired synchronization with stronger robustness and less power. It does satisfy two important issues in the synchronization of neurons with external electrical stimulations.

\section{Conclusion}

In this paper, LADRC is adopted to discuss the synchronization of Morris-Lecar neuronal membrane potentials. Theoretically, it has been analysed that LADRC can get asymptotic synchronization of neuronal membrane potentials by selecting appropriate parameters of ESO and controller. Meanwhile, from numerical results, it has been verified that LADRC can perform well in the synchronization, no matter disturbance and (or) uncertainties exist or not.

Precise model is not necessary and less control energy is needed, both of the facts guarantee that LADRC is able to achieve better synchronization. Broader prospects in practical synchronization of membrane potentials in nervous systems can be expected.

\section{Data Availability}

The data used to support the findings of this study are available from the corresponding author upon request.

\section{Disclosure}

Some of the content has been discussed on an academic conference.

\section{Conflicts of Interest}

The authors declare that there are no conflicts of interest regarding the publication of this paper.

\section{Acknowledgments}

This work is supported by Project of High-level Teachers in Beijing Municipal Universities in the Period of 13th FiveYear Plan (CIT\&TCD201704044), National Natural Science Foundation of China (61403006), Key program of Beijing Municipal Education Commission (KZ201810011012), and the 


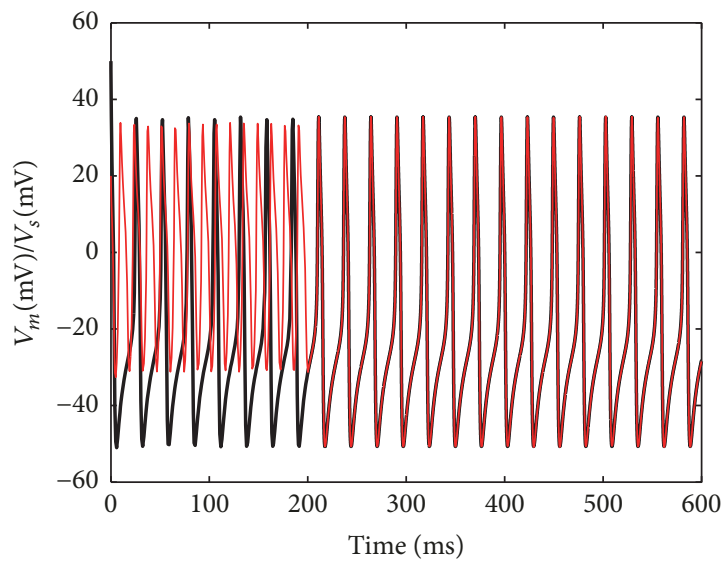

$-V_{m}(\mathrm{mV})$

$-V_{s}(\mathrm{mV})$

(a)

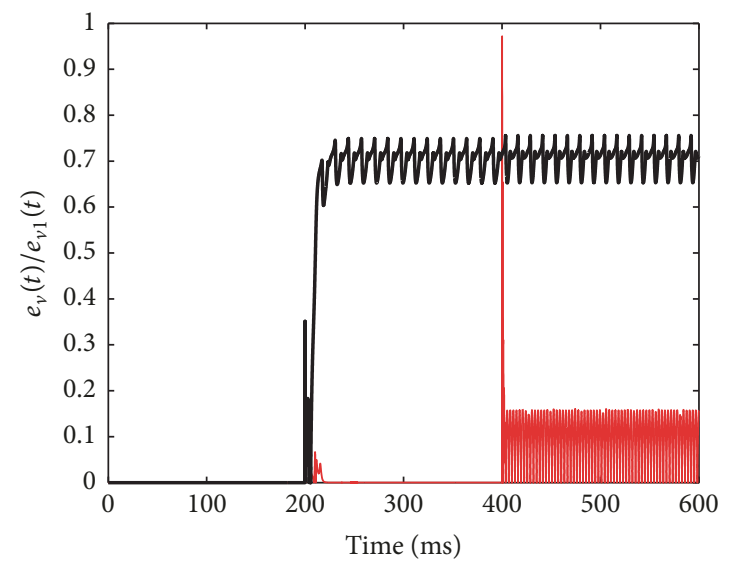

$-e_{v}(t)$

$-e_{v 1}(t)$

(c)

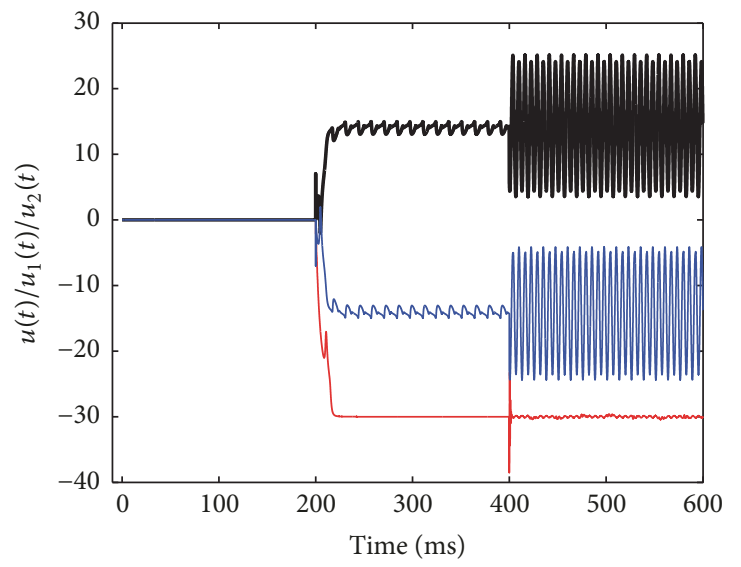

$u(t)$
$-\quad u_{1}(t)$
$-u_{2}(t)$

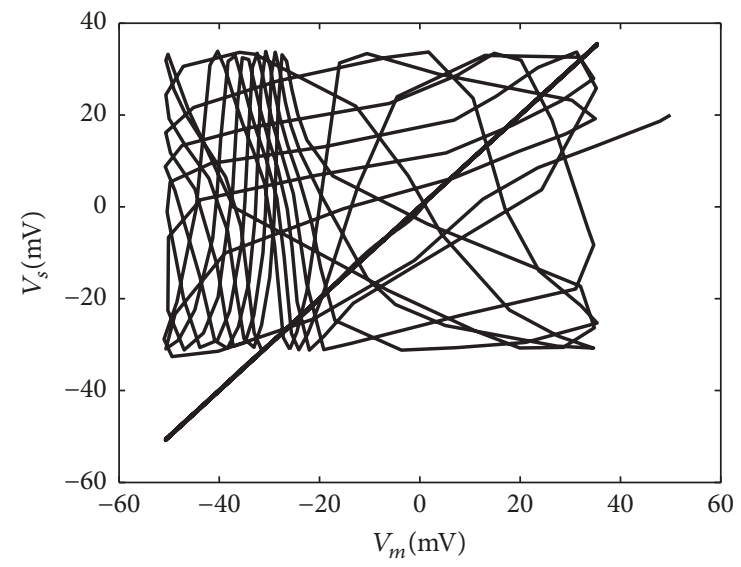

(b)



$-e_{n}(t)$
$-e_{n 1}(t)$

(d)

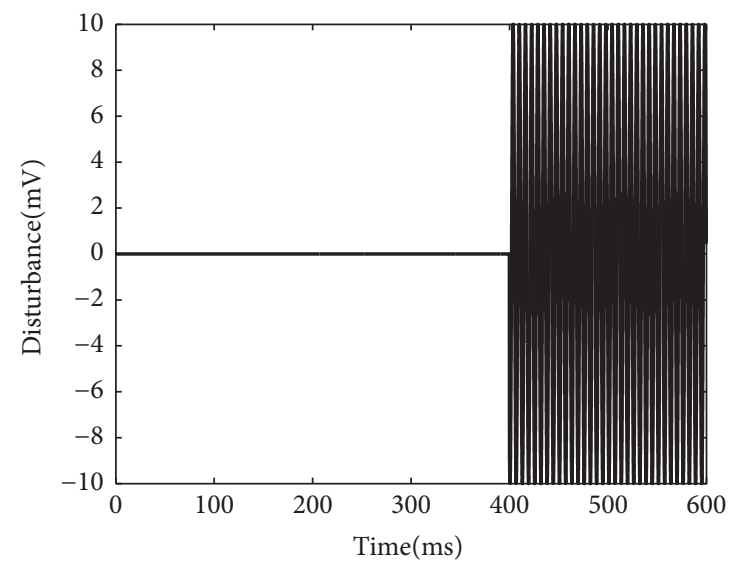

(f)

FIGURE 4: Synchronous response of Morris-Lecar neurons and the comparisons of control inputs (in presence of sinusoidal disturbance). 


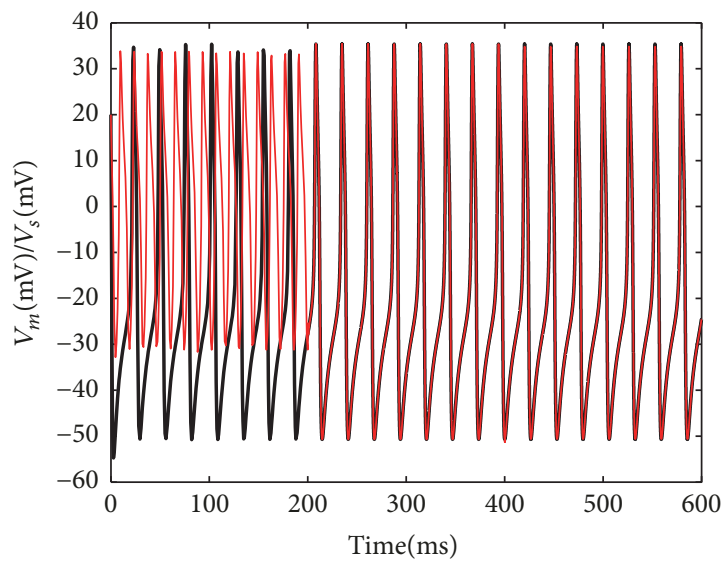

$-V_{m}(\mathrm{mV})$
$-V_{s}(\mathrm{mV})$

(a)

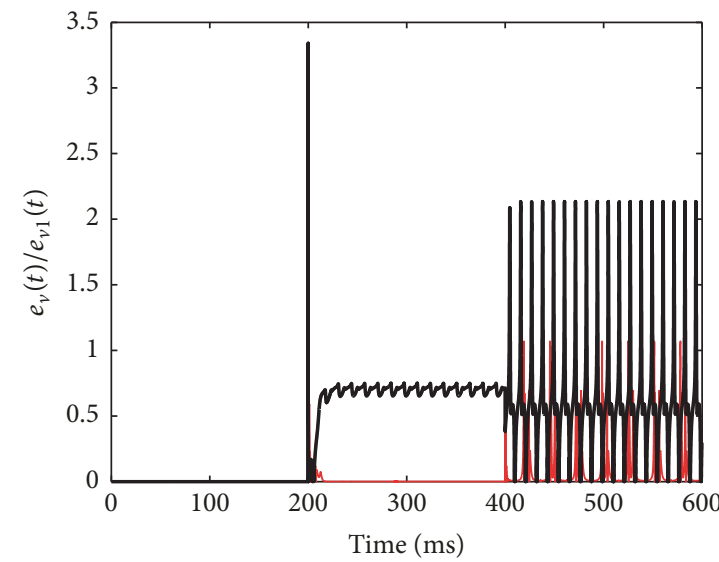

$-e_{v}(t)$

$e_{v 1}(t)$

(c)

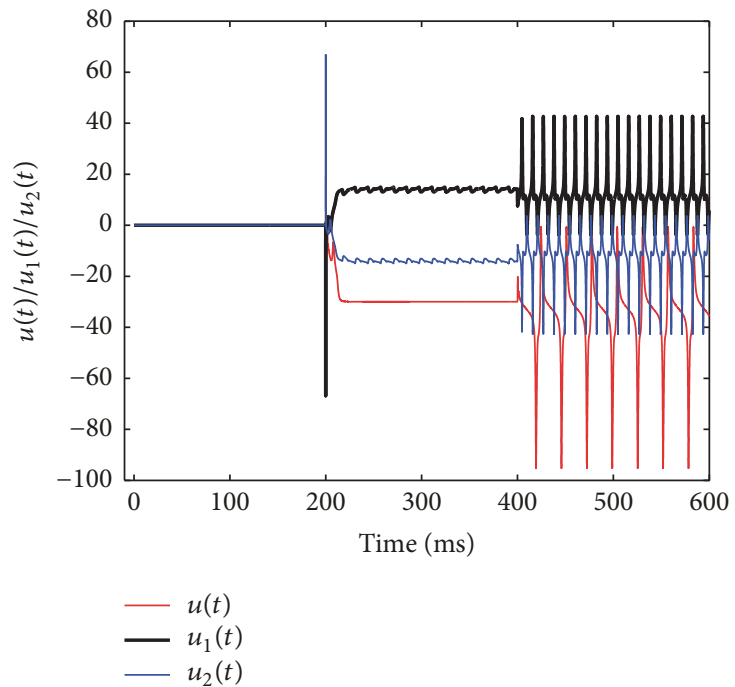

(e)

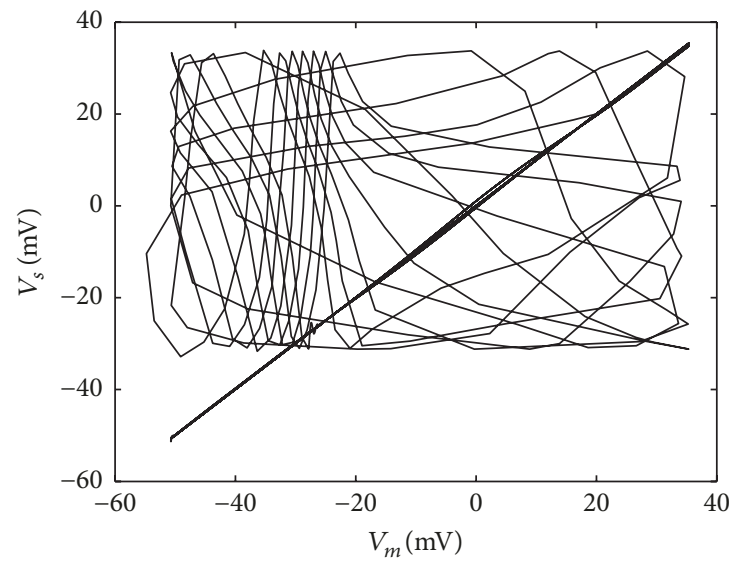

(b)

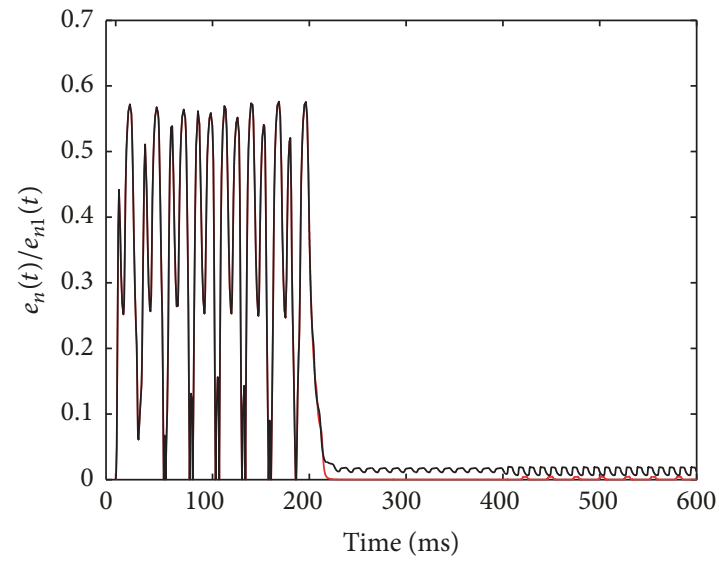

$-e_{n}(t)$

$-e_{n}(t)$
$-e_{n 1}(t)$

(d)

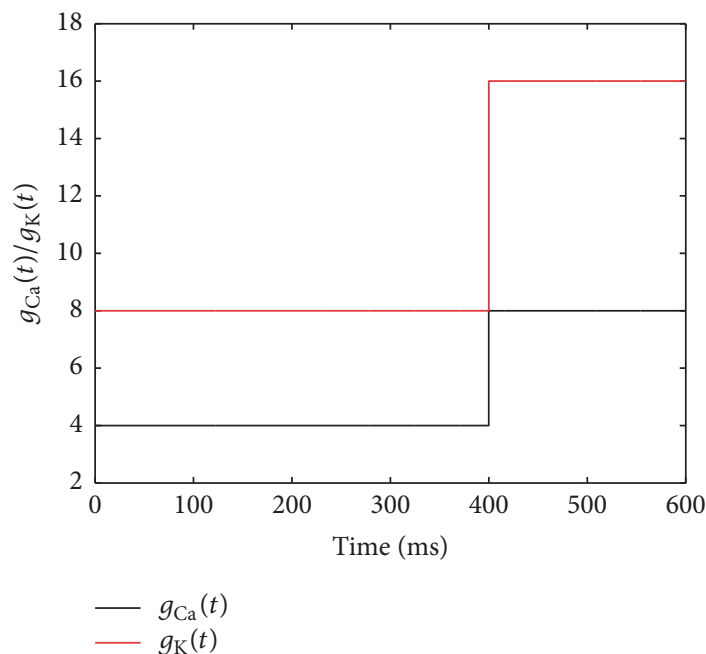

(f)

FIGURE 5: Synchronous response of Morris-Lecar neurons and the comparisons of control inputs (in presence of parameter perturbations). 
National Key Research and Development Program of China (2016YFD0401205).

\section{References}

[1] T. Womelsdorf and P. Fries, "The role of neuronal synchronization in selective attention," Current Opinion in Neurobiology, vol. 17, no. 2, pp. 154-160, 2007.

[2] A. L. Hodgkin and A. F. Huxley, "A quantitative description of membrane current and its application to conduction and excitation in nerve.," The Journal of Physiology, vol. 117, no. 4, pp. 500$544,1952$.

[3] R. Fitzhugh, "Thresholds and plateaus in the Hodgkin-Huxley nerve equations," The Journal of General Physiology, vol. 43, pp. 867-896, 1960.

[4] B. Doiron, C. Laing, A. Longtin, and L. Maler, "Ghostbursting: A novel neuronal burst mechanism," Journal of Computational Neuroscience, vol. 12, no. 1, pp. 5-25, 2002.

[5] J. L. Hindmarsh and R. M. Rose, "A model of neuronal bursting using three coupled first order differential equations," Proceedings of the Royal Society of London B: Biological Sciences, vol. 221, no. 1222, pp. 87-102, 1984.

[6] T. R. Chay, "Chaos in a three-variable model of an excitable cell," Physica D: Nonlinear Phenomena, vol. 16, no. 2, pp. 233-242, 1985.

[7] O. Comejo-Pérez and R. Femat, "Unidirectional synchronization of Hodgkin-HUXley neurons," Chaos, Solitons \& Fractals, vol. 25, no. 1, pp. 43-53, 2005.

[8] L. H. Nguyen and K.-S. Hong, "Adaptive synchronization of two coupled chaotic Hindmarsh-Rose neurons by controlling the membrane potential of a slave neuron," Applied Mathematical Modelling: Simulation and Computation for Engineering and Environmental Systems, vol. 37, no. 4, pp. 2460-2468, 2013.

[9] L. H. Nguyen and K.-S. Hong, "Synchronization of coupled chaotic FitzHugh-Nagumo neurons via Lyapunov functions," Mathematics and Computers in Simulation, vol. 82, no. 4, pp. 590-603, 2011.

[10] H. Y. Li, Y. K. Wong, W. L. Chan, and K. M. Tsang, "Synchronization of Ghostburster neurons under external electrical stimulation via adaptive neural network $\mathrm{H}$ control," Neurocomputing, vol. 74, no. 1-3, pp. 230-238, 2010.

[11] J. Wang, M. Lu, X. Ye, and X. Fei, "Asymptotic behavior of Morris-Lecar system," Nonlinear Analysis: Real World Applications, vol. 9, no. 3, pp. 852-857, 2008.

[12] J. Wang, M. Lu, and H. Li, "Synchronization of coupled equations of Morris-Lecar model," Communications in Nonlinear Science and Numerical Simulation, vol. 13, no. 6, pp. 1169-1179, 2008.

[13] J. M. González-Miranda, "Pacemaker dynamics in the full Morris-Lecar model," Communications in Nonlinear Science and Numerical Simulation, vol. 19, no. 9, pp. 3229-3241, 2014.

[14] M. Shi and Z. Wang, "Abundant bursting patterns of a fractional-order Morris-Lecar neuron model," Communications in Nonlinear Science and Numerical Simulation, vol. 19, no. 6, pp. 1956-1969, 2014.

[15] Y.-Y. Chen, J. Wang, Y.-Q. Che, X.-L. Wei, and B. Deng, "Unidirectional synchronization of Morris-Lecar neurons via adaptive $\mathrm{H} \infty$ control," in Proceedings of the 29th Chinese Control Conference, CCC'10, pp. 749-754, Beijing, China, July 2010.

[16] X. Lou and M. N. S. Swamy, "A new approach to optimal control of conductance-based spiking neurons," Neural Networks, vol. 96, pp. 128-136, 2017.
[17] J. Q. Han, "Control theory—a model analysis approach or a direct control approach?" Journal of Systems Science and Mathematical Sciences, vol. 9, no. 4, pp. 328-335, 1989.

[18] J. Q. Han, "Active disturbance rejection controller and its application," Control and Decision-making, vol. 13, no. 1, pp. 19-23, 1998.

[19] Y. Jiang, Q. Sun, X. Zhang, and Z. Chen, "Pressure regulation for oxygen mask based on active disturbance rejection control," IEEE Transactions on Industrial Electronics, vol. 64, no. 8, pp. 6402-6411, 2017.

[20] C. Dai, J. Yang, Z. Wang, and S. Li, "Universal active disturbance rejection control for non-linear systems with multiple disturbances via a high-order sliding mode observer," IET Control Theory \& Applications, vol. 11, no. 8, pp. 1194-1204, 2017.

[21] S. Shao and Z. Gao, "On the conditions of exponential stability in active disturbance rejection control based on singular perturbation analysis," International Journal of Control, vol. 90, no. 10, pp. 2085-2097, 2017.

[22] Z. Gao, "Scaling and bandwidth-parameterization based controller tuning," in Proceedings of the American Control Conference, pp. 4989-4996, Denver, Colo, USA, June 2003.

[23] Z. Q. Chen, M. W. Sun, and R. G. Yang, "Stability of linear active disturbance rejection control," Acta Automatica Sinica, vol. 39, no. 5, pp. 574-580, 2013. 


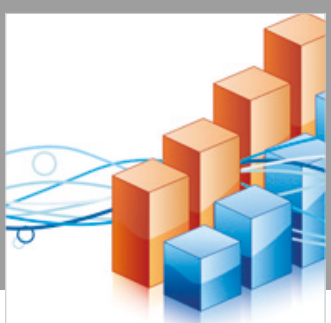

Advances in

Operations Research

\section{-n-m}
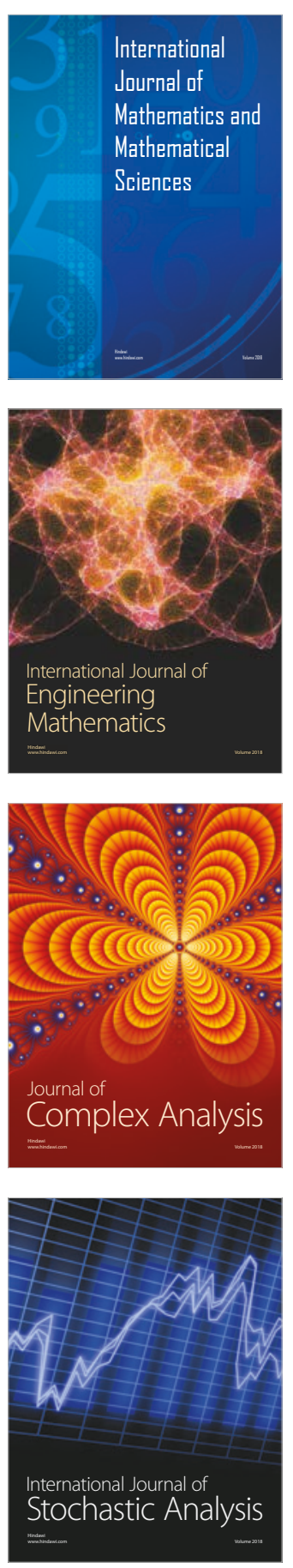
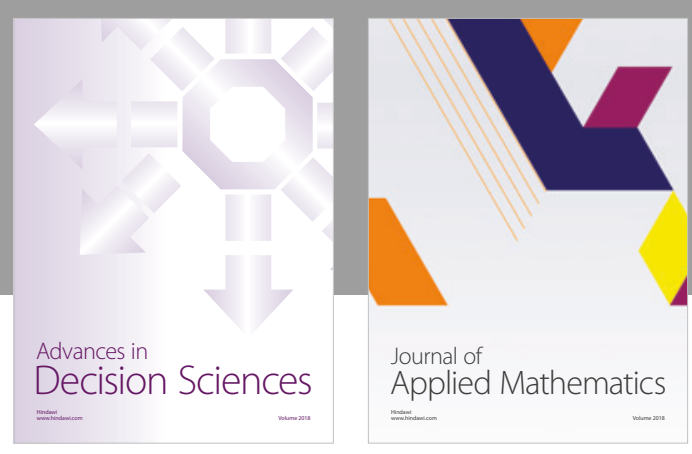

Journal of

Applied Mathematics
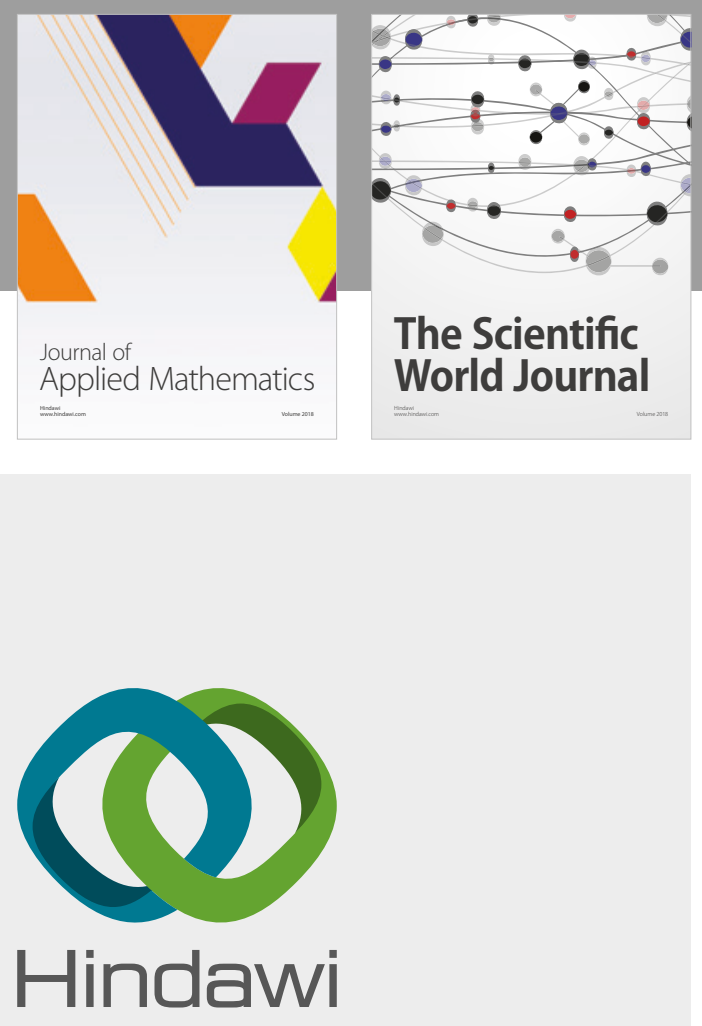

Submit your manuscripts at

www.hindawi.com

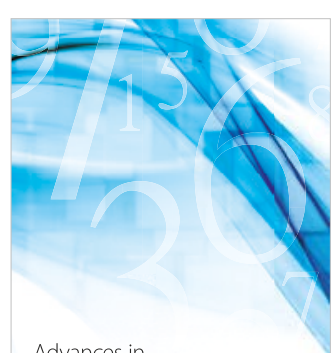

Advances in
Numerical Analysis
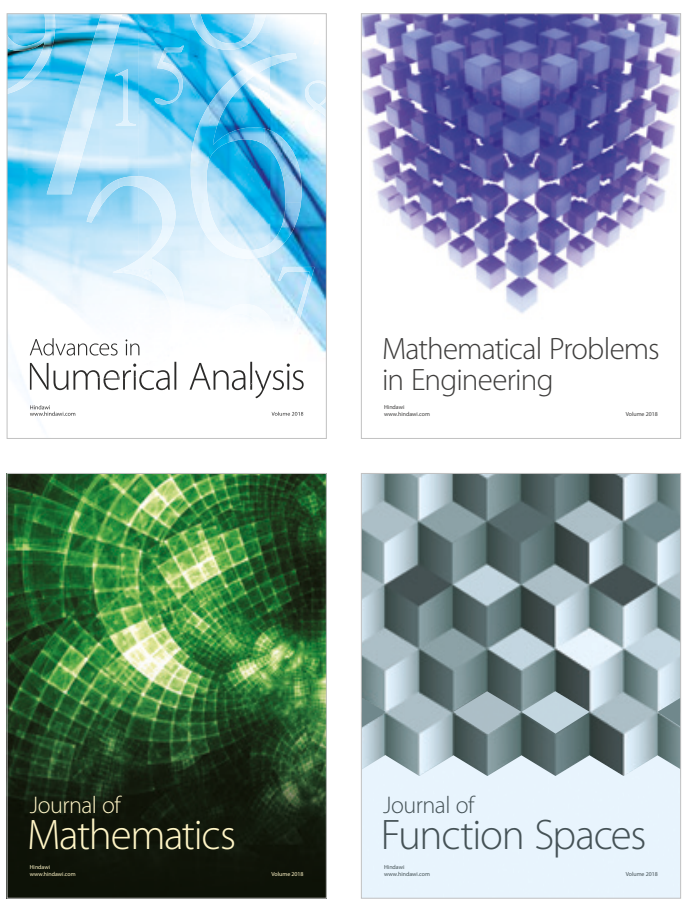

Mathematical Problems in Engineering

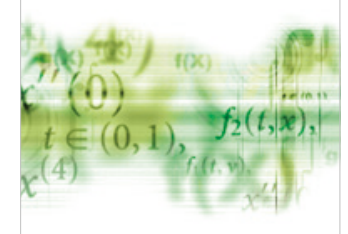

International Journal of

Differential Equations

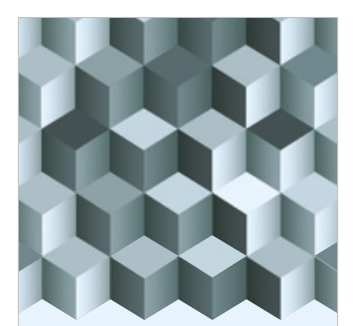

Journal of

Function Spaces
The Scientific

World Journal



Journal of

Probability and Statistics
